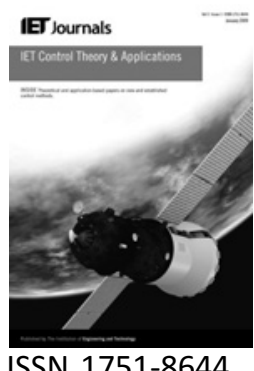

\title{
New insight into internal model control filter design for load disturbance rejection
}

\section{T. Liu F. Gao}

\author{
Department of Chemical \& Biomolecular Engineering, Hong Kong University of Science \& Technology, Clear Water Bay, \\ Kowloon, Hong Kong \\ E-mail: kefgao@ust.hk
}

\begin{abstract}
In this study, a modified design of the internal model control (IMC) filter is proposed for improving closed-loop system performance of load disturbance rejection, especially for slow processes in industrial and chemical engineering practices. The deficiency of a conventional IMC filter design for controller tuning is revealed with regard to load disturbance rejection. By constructing one or more asymptotic canceling constraints for disturbance rejection, a modified IMC filter is proposed to reduce the influence from the time constant(s) of the process or repetitive-type load disturbance to the closed-loop disturbance rejection performance. Similar to a conventional IMC filter, there is essentially a single adjustable parameter in the proposed IMC filter, which can be monotonically tuned to meet with the compromise between the achievable disturbance rejection performance and the closed-loop system stability. Quantitative tuning formulae and guidelines for this adjustable parameter are developed in terms of the widely used first- and second-order process models with time delay. Illustrative examples are given to show the effectiveness and merits of the proposed IMC filter.
\end{abstract}

\section{Introduction}

Load disturbance rejection is one of the most important issues in the context of process control. Internal model control (IMC) design [1] has been widely recognised and applied for closed-loop disturbance rejection in engineering practices [2-4]. A large number of controller tuning methods based on the unity feedback control structure have been well developed in terms of the IMC principle, leading to superior disturbance rejection performance as compared with other tuning methods. To enumerate a few, earlier literature [5] presented simple IMC tuning formulae for low-order industrial processes; a unified IMC-based PID or high-order controller design was proposed [6] in terms of the standard/recursive least-squares (LS) fitting algorithm to achieve a desired closed-loop response specification; [7-10] developed analytical PI/PID tuning formulae for a variety of linear time-invariant process models by means of the Taylor series or Padé approximation; according to the integral-square-error (ISE) criterion, two numerical optimisation methods were recently presented $[11,12]$ to refine some of the aforementioned IMC tuning formulae for industrial processes with time delay. Based on the standard IMC structure, Liu et al. [13] developed an analytical decoupling control method for multivariable processes with time delays, obtaining improved disturbance rejection performance compared to other decoupling control methods. To separately optimise system performance for load disturbance rejection without sacrificing the nominal set-point tracking performance, the standard two-degreeof-freedom (2DOF) IMC structure shown in Fig. 1 can be utilised [1]. Tian and Gao [14] demonstrated that such a 2DOF control structure is particularly effective for chemical processes with dominant time delay. It can be verified, by comparing the transfer functions respectively for set-point tracking and load disturbance rejection, that the standard 2DOF IMC structure with a conventional IMC filter of type I prescribed for the two controllers is capable of the same closed-loop system performance with $[15,16]$, both of which had shown their superiority of load disturbance rejection over many other control methods. 


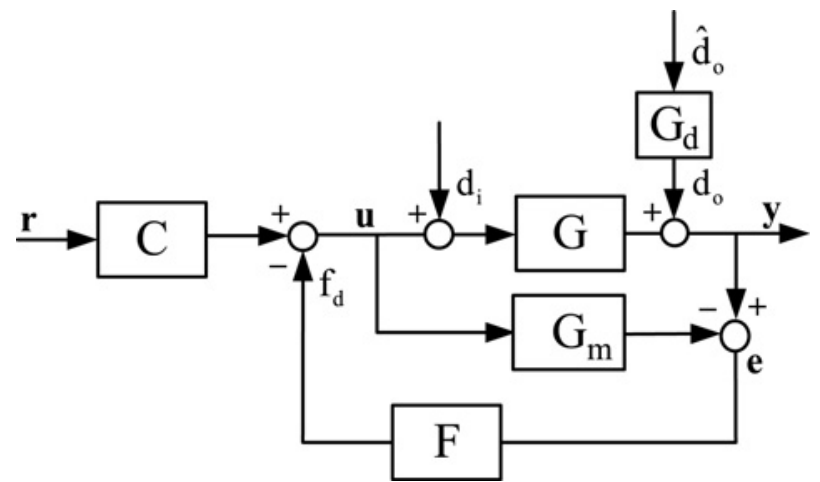

Figure 1 Two-degree-of-freedom IMC structure

The key to IMC-based controller design lies with the choice of a suitable IMC filter to prescribe a desired closed-loop complementary sensitivity function. Given a process model $G_{\mathrm{m}}=G_{\mathrm{A}} G_{\mathrm{M}}$, where $G_{\mathrm{A}}$ is an all-pass portion and $G_{\mathrm{M}}$ a minimum-phase portion, the IMCbased complementary sensitivity function can be determined as $T=G_{\mathrm{A}} f$, where $f$ denotes the IMC filter [17]. A conventional IMC filter of type I, $f(s)=1 /(\lambda s+1)^{n}$, is generally chosen for step change in set-point or load disturbance, and type II, $f(s)=(n \lambda s+1) /(\lambda s+1)^{n}$, is for ramp change, where $n$ is an integer large enough to ensure $f / G_{\mathrm{M}}$ proper. Most of IMC-based controller tuning methods, including the aforementioned, are based on the filter type I, since a step change in set-point or load disturbance can be physically regarded as a summation of sinusoidal signals of different frequencies. The key feature of IMC filter type I is that it can lead to the $\mathrm{H}_{2}$ optimal performance objective [1], $\min \|e\|_{2}^{2}$, for set-point tracking and step change of the load disturbance acting on the process output side (denoted as $d_{\mathrm{o}}$ in Fig. 1). However, for a load disturbance seeping into the process, denoted as $d_{\mathrm{i}}$ in Fig. 1, the corresponding transfer function is $H_{d_{\mathrm{i}}}=G S d_{\mathrm{i}}$, where $S=1-T$ is the closed-loop sensitivity function. It can be seen that the time constant(s) of $G$ is enclosed in the characteristic equation of $H_{d i}$, and therefore, affects the achievable disturbance rejection performance, no matter how the IMC filter is tuned in $T$. For a slow process with a large time constant, the recovery trajectory of the disturbance response is subject to 'a long tail' (i.e. sluggish load disturbance suppression), as early reported by Horn et al. [18]. Besides, if there exists a load disturbance transfer function, $G_{\mathrm{d}}$, as shown in Fig. 1, the achievable disturbance rejection performance is also restricted by the time constant(s) of $G_{\mathrm{d}}$. In many industrial cases, $G_{\mathrm{d}}$ has a similar form to $G$, so the influence of $\hat{d}_{\mathrm{o}}$ may be transformed into $d_{\mathrm{i}}$ to be treated with [4].

Using an idea of eliminating the slowest pole of $G$, Horn et al. [18] suggested a improved IMC filter design for some delay-free processes, and first-order processes with small time delay that can be properly approximated by the firstorder Padé expansion. Further extended PID tuning methods were recently presented for improving disturbance rejection [19]. Middleton and Graebe [20] analysed the necessity of canceling the slow stable pole(s) of a delay-free process for controller design in terms of the unity feedback control structure. Campi et al. [21] presented another type of IMC filter for industrial systems with an unstable pole, with two adjustable parameters respectively for regulating the closed-loop bandwidth and the recovery time.

In this paper, by establishing asymptotic canceling constraints to reduce the influence from the time constant(s) of the process or repetitive-type load disturbance, a modified IMC filter design is proposed to improve disturbance rejection performance for slow processes with time delay. Quantitative tuning formulae and guidelines are developed in terms of the widely used first- and second-order process models with time delay. The closed-loop structure for disturbance rejection in the standard 2DOF IMC structure shown in Fig. 1 is adopted to present the proposed IMC filter design, where the feedback controller, $F$, is to be configured with the proposed IMC filter. Note that, by using the equivalent transform between the standard IMC structure and the conventional unity feedback control loop [1], the proposed IMC filter can also be applied to tune the loop controller for load disturbance rejection. The paper is organised as follows: Section 2 presents the proposed IMC filter design based on the widely used first- and secondorder process models with time delay, together with some discussions on the achievable disturbance rejection performance. In Section 3, robust stability constraints associated with tuning the proposed IMC filter are analysed for the presence of process uncertainties. Illustrative examples are given in Section 4 to demonstrate the effectiveness and merits of the proposed IMC filter. Finally, conclusions are drawn in Section 5.

\section{Proposed IMC filter design}

It can be seen from Fig. 1 that in the nominal case, $G=G_{\mathrm{m}}$, the transfer function of load disturbance response relating $d_{\mathrm{i}}$ to $y$ can be derived as

$$
\frac{y_{\mathrm{di}}}{d_{\mathrm{i}}}=G\left(1-F G_{\mathrm{m}}\right)
$$

Note that the nominal closed-loop complementary sensitivity function can be formulated as $T=F G$, which is actually equivalent to the transfer function from the load disturbance $d_{\mathrm{i}}$ to the controller output $f_{\mathrm{d}}$. Therefore (1) can be rewritten as

$$
\frac{y_{\mathrm{di}}}{d_{\mathrm{i}}}=G(1-T)
$$

To reduce the influence arising from the process time constant(s) to the load disturbance response, it is of course ideal to eliminate the corresponding pole(s) from the characteristic equation of (2). It is thus expected that $1-T$ 
(i.e. $S$ ), rather than $T$, has the corresponding zero(s) to cancel the pole(s) of $G$, such that the characteristic equation is governed only by the time constant of $T$ (i.e. an adjustable parameter in the IMC filter). The numerator of $1-T$, however, is unavoidably involved with time delay factor(s) for a process with time delay, so it cannot be analytically factorised to make zero-pole cancellation with the denominator of $G$. The following asymptotic constraint(s) is therefore proposed to realise the above idea

$$
\lim _{s \rightarrow-p_{i}}(1-T)=0, \quad i=1,2, \ldots, m
$$

where $p_{i}$ is the reciprocal of the process time constant(s).

Based on the widely used first- and second-order process models with time delay, the proposed IMC filter design is detailed in the following two subsections.

\subsection{IMC filter for first-order process}

A first-order process is generally modelled as

$$
G_{1}=\frac{k}{\tau s+1} \mathrm{e}^{-\theta s}
$$

where $k$ is the proportional gain, $\tau$ is the time constant and $\theta$ is the process time delay.

According to the IMC theory, the conventional IMC filter should be configured as

$$
f_{\mathrm{IMC}-1}=\frac{1}{\lambda s+1}
$$

where $\lambda$ is a user specified time constant, that is, an adjustable parameter. Correspondingly, the nominal closed-loop complementary sensitivity function is obtained as

$$
T_{\mathrm{IMC}-1}=\frac{\mathrm{e}^{-\theta s}}{\lambda s+1}
$$

To improve the load disturbance response, the conventional IMC filter in (6) is hereby rectified as

$$
f_{\mathrm{RIMC}-1}=\frac{\alpha s+1}{\left(\lambda_{\mathrm{f}} s+1\right)^{2}}
$$

where $\alpha$ is an additional parameter used to satisfy the following asymptotic constraint

$$
\lim _{s \rightarrow-1 / \tau}(1-T)=0
$$

Accordingly, it follows that

$$
T_{\mathrm{RIMC}-1}=\frac{(\alpha s+1) \mathrm{e}^{-\theta s}}{\left(\lambda_{\mathrm{f}} s+1\right)^{2}}
$$

Substituting (9) into (8) yields

$$
\alpha=\tau\left[1-\left(\frac{\lambda_{\mathrm{f}}}{\tau}-1\right)^{2} \mathrm{e}^{-\theta / \tau}\right]
$$

It is thus seen that $\alpha$ is a function of $\lambda_{\mathrm{f}}$. So, there is essentially a single adjustable parameter, $\lambda_{f}$, in the proposed IMC filter.

Using the nominal closed-loop relationship, $T=F G$, we obtain the feedback controller

$$
F_{\mathrm{RIMC}-1}=\frac{(\alpha s+1)(\tau s+1)}{k\left(\lambda_{\mathrm{f}} s+1\right)^{2}}
$$

Remark 1: Note that $F_{\mathrm{RIMC}-1}=1 / k$ when $\lambda_{\mathrm{f}}$ is tuned as $\tau$ (or $\tau_{\mathrm{d}}$ of $G_{d}$ ), for which $T$ becomes the same as $G$ (or $G_{\mathrm{d}}$ ). When $\lambda_{\mathrm{f}}$ is tuned larger than $\tau$ (or $\tau_{\mathrm{d}}$ ), the load disturbance response will be slower than $G$ (or $G_{\mathrm{d}}$ ). Hence, it is suggested to tune $\lambda_{\mathrm{f}}<\tau$ for load disturbance rejection, unless it is intentionally violated to obtain sustainable closed-loop stability in order to accommodate for process uncertainties.

By substituting (9) into (2) and taking the inverse Laplace transform, the time response for a step change of $d_{\mathrm{i}}$ can be derived as (see (12))

Note that $y_{\mathrm{d}_{\mathrm{i}}}(t)$ increases monotonically in the time interval $t \in(\theta, 2 \theta]$ and $\mathrm{d} y_{\mathrm{d}_{\mathrm{i}}}(t) /\left.\mathrm{d} t\right|_{t=2 \theta} \neq 0$. Its peak value should be reached in the time interval $(2 \theta, \infty)$. The time to reach the disturbance response peak (DP) can be derived by solving $\mathrm{d} y_{\mathrm{d}_{\mathrm{i}}}(t) / \mathrm{d} t=0$ for the final phase as

$$
t_{\mathrm{DP}}=2 \theta+\frac{\lambda_{\mathrm{f}}^{2} \mathrm{e}^{-\theta / \tau}}{\tau+\left(\lambda_{\mathrm{f}}-\tau\right) \mathrm{e}^{-\theta / \tau}}
$$

Substituting (13) into (12), we obtain

$$
\begin{aligned}
y_{\mathrm{d}_{\mathrm{i}}}\left(t_{\mathrm{DP}}\right)= & k\left[1+\left(\frac{\lambda_{\mathrm{f}}}{\tau}-1\right) \mathrm{e}^{-\theta / \tau}\right] \\
& \times \mathrm{e}^{-\left(\left(\lambda_{\mathrm{f}} / \tau\right) \mathrm{e}^{(-\theta / \tau)}\right) / 1+\left(\lambda_{\mathrm{f}} / \tau-1\right) \mathrm{e}^{-(\theta / \tau)}}
\end{aligned}
$$

To make clear the tuning relationship between the adjustable parameter $\lambda_{\mathrm{f}}$ and DP, the following proposition is given.

$$
y_{\mathrm{d}_{\mathrm{i}}}(t)= \begin{cases}0, & t \leq \theta ; \\ k\left(1-e^{-[t-\theta / \tau]}\right), & \theta<t \leq 2 \theta ; \\ k\left[1-e^{-(\theta / \tau)}+\frac{1+\left[\left(\lambda_{\mathrm{f}} / \tau\right)-1\right] e^{-(\theta / \tau)}}{\lambda_{\mathrm{f}}}(t-2 \theta)\right] e^{-\left[(t-2 \theta) / \lambda_{\mathrm{f}}\right]}, & t>2 \theta\end{cases}
$$


Proposition 1: For a first-order process, DP of the load disturbance response $\left(y_{\mathrm{di}}\right)$ increases monotonically with respect to $\lambda_{f}$.

Proof: Letting $A=1+\left(\lambda_{\mathrm{f}} / \tau-1\right) \mathrm{e}^{-\theta / \tau}$ and $B=1-e^{-\theta / \tau}$, the first derivative of $y_{\mathrm{d}_{\mathrm{i}}}\left(t_{\mathrm{DP}}\right)$ shown in (14) can be derived as

$$
\frac{\mathrm{d} y_{\mathrm{d}_{\mathrm{i}}}\left(t_{\mathrm{DP}}\right)}{\mathrm{d} \lambda_{\mathrm{f}}}=\frac{k}{\tau} \mathrm{e}^{(B / A)-(\theta / \tau)-1}\left(1-\frac{B}{A}\right)
$$

Owing to the fact that $A>B>0$, there exists $\mathrm{d} y_{\mathrm{d}_{\mathrm{i}}}\left(t_{\mathrm{DP}}\right) / \mathrm{d} \lambda_{\mathrm{f}}>0$. Hence, it follows the conclusion in Proposition 1.

Note that the above analysis of DP may be utilised to assess the maximum deviation from the set-point value for the presence of load disturbance in engineering practices.

Define the recovery time, $t_{\mathrm{re}}$, as the period from the moment that a step change of load disturbance is added to the process to the moment that the load disturbance response recovers into the $5 \%$ error band that is usually specified for set-point tracking in practice. It follows from (12) that

$$
1-\mathrm{e}^{-\theta / \tau}+\frac{t_{\mathrm{re}}-2 \theta}{\lambda_{\mathrm{f}}}\left[1+\left(\frac{\lambda_{\mathrm{f}}}{\tau}-1\right) e^{-\theta / \tau}\right]=0.05 \mathrm{e}^{\left(t_{\mathrm{re}}-2 \theta\right) / \lambda_{\mathrm{f}}}
$$

Apparently, (15) is a transcendental equation that cannot be solved analytically. Numerical computation in terms of the Newton-Raphson algorithm is therefore cultivated to disclose the quantitative tuning relationship between $\lambda_{\mathrm{f}}$ and $t_{\mathrm{re}}$. By sweeping over the ratio regions of $\lambda_{\mathrm{f}} / \tau \in[0.1,2.0]$ and $\theta / \tau \in[0.1,2.0]$, the numerical results of scaled recovery time, $t_{\text {re }} / k$, are plotted in Fig. 2 . Note that $\tau=1$ is assumed to obtain the scaled recovery time shown in Fig. 2. Given $\tau \neq 1$, the recovery time can be graphically read as $t_{\mathrm{re}}=\left.\tau t_{\mathrm{re}}\right|_{\tau=1}$, in view of that $t_{\mathrm{re}} / \tau$ and $\left.t_{\mathrm{re}}\right|_{\tau=1}$ correspond to the identical solution of $\lambda_{\mathrm{f}} / \tau$ for (15).

Remark 2: Although the mathematical Lambert W function, defined as the multi-inverse function for $\omega \mapsto \omega \mathrm{e}^{\omega}$, may be employed to solve (15) to yield an 'analytical' expression, $t_{\mathrm{re}}=2 \theta+\lambda_{\mathrm{f}}\left[-W\left(-\mathrm{e}^{-A / B} / B\right)\right.$ $-A / B]$, where $A=20\left(1-e^{-\theta / \tau}\right)$ and $B=20[1+$ $\left.\left(\lambda_{\mathrm{f}} / \tau-1\right) \mathrm{e}^{-\theta / \tau}\right]$, it may lead to an incorrect result because the principle branch value of the Lambert $\mathrm{W}$ function is generally yielded in commercial symbolic software packages [22]. Note that, in the above expression $-W\left(-\mathrm{e}^{-A / B} / B\right)-A / B$ should be positive since $t_{\mathrm{re}}>2 \theta$, but it may give a negative value because $W\left(-\mathrm{e}^{-A / B} / B\right)>W\left(-A e^{-A / B} / B\right)=-A / B$ is yielded from the principle branch of the Lambert $W$ function. According to the numerical results given in Fig. 2, the secondary real branch of the Lambert $\mathrm{W}$ function should be used to compute $t_{\mathrm{re}}$ from the above expression.

\subsection{IMC filter for second-order process}

A second-order process is generally described as

$$
G_{2}=\frac{k \omega_{\mathrm{n}}^{2}}{s^{2}+2 \xi \omega_{\mathrm{n}} s+\omega_{\mathrm{n}}^{2}} \mathrm{e}^{-\theta s}
$$

where $\omega_{\mathrm{n}}$ denotes the natural frequency and $\xi$ the damping ratio.

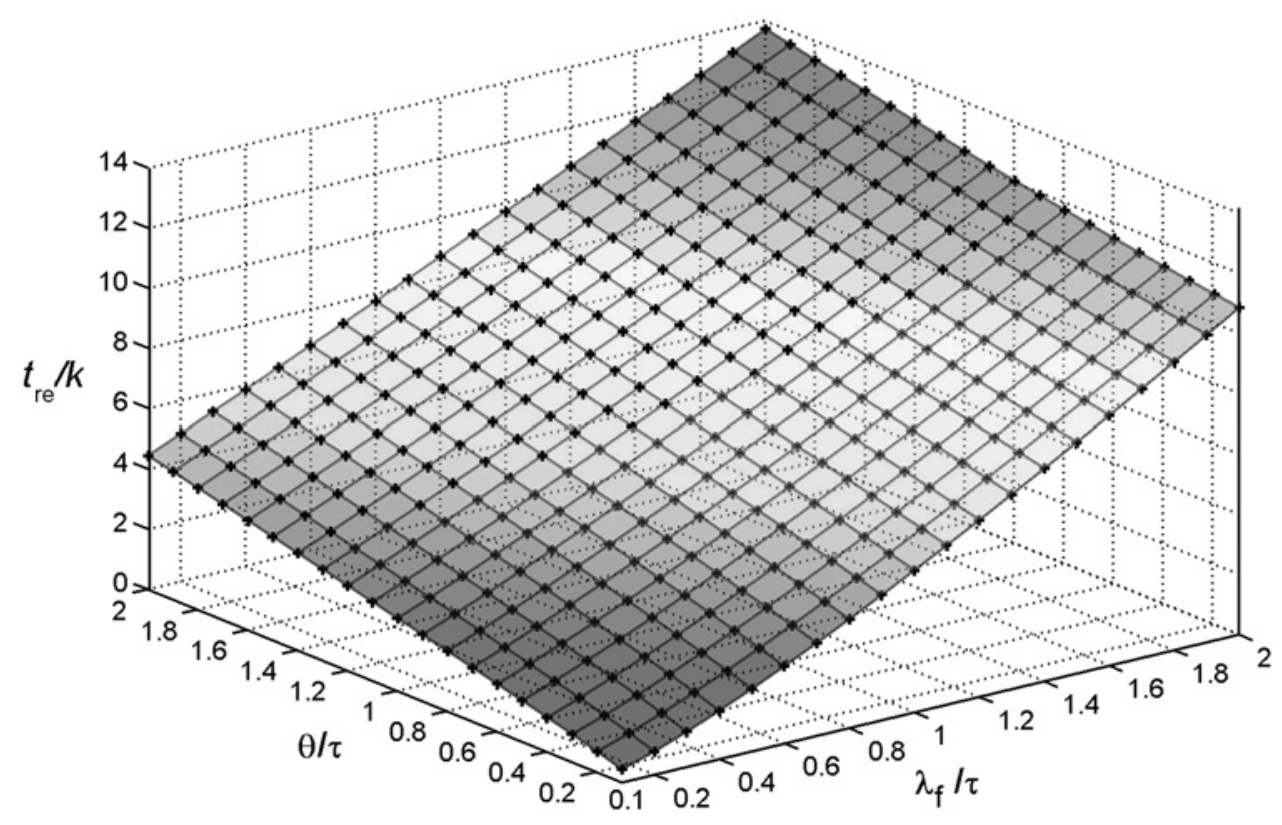

Figure 2 Recovery time for a first-order process 
According to the IMC theory, the conventional IMC filter should be configured as

$$
f_{\mathrm{IMC}-2}=\frac{1}{(\lambda s+1)^{2}}
$$

To improve the load disturbance response, the conventional IMC filter shown in (17) is rectified as

$$
f_{\mathrm{RIMC}-2}=\frac{\alpha s^{2}+\beta s+1}{\left(\lambda_{\mathrm{f}} s+1\right)^{4}}
$$

where $\alpha$ and $\beta$ are utilised to satisfy the following asymptotic constraints

$$
\begin{aligned}
& \lim _{s \rightarrow-p_{1}}(1-T)=0 \\
& \lim _{s \rightarrow-p_{2}}(1-T)=0
\end{aligned}
$$

where

$$
\begin{aligned}
& p_{1}=\left\{\begin{array}{ll}
\omega_{\mathrm{n}}\left(\xi-j \sqrt{1-\xi^{2}}\right), & 0<\xi<1 \\
\omega_{\mathrm{n}}\left(\xi-\sqrt{\xi^{2}-1}\right), & \xi \geq 1
\end{array},\right. \\
& p_{2}= \begin{cases}\omega_{\mathrm{n}}\left(\xi+j \sqrt{1-\xi^{2}}\right), & 0<\xi<1 \\
\omega_{\mathrm{n}}\left(\xi+\sqrt{\xi^{2}-1}\right), & \xi \geq 1\end{cases}
\end{aligned}
$$

Note that $-p_{1}$ and $-p_{2}$ are the two poles of $G_{2}$. When $\xi=1$, there exists $p_{1}=p_{2}=\omega_{\mathrm{n}}$, so (19) becomes the same as (20). Another asymptotic constraint should therefore be imposed to derive $\alpha$ and $\beta$, that is

$$
\lim _{s \rightarrow-p_{1}} \frac{\mathrm{d}}{\mathrm{d} s}(1-T)=0
$$

Accordingly, the nominal closed-loop complementary sensitivity function can be derived as

$$
T_{\mathrm{RIMC}-2}=\frac{\left(\alpha s^{2}+\beta s+1\right) \mathrm{e}^{-\theta s}}{\left(\lambda_{\mathrm{f}} s+1\right)^{4}}
$$

When $\xi \neq 1$, by substituting (22) into (19) and (20), respectively, we obtain

$$
\begin{aligned}
& \alpha=\frac{p_{1} \mathrm{e}^{-\theta p_{2}}\left(p_{2} \lambda_{\mathrm{f}}-1\right)^{4}-p_{2} \mathrm{e}^{-\theta p_{1}}\left(p_{1} \lambda_{\mathrm{f}}-1\right)^{4}-p_{1}+p_{2}}{p_{1} p_{2}\left(p_{2}-p_{1}\right)} \\
& \beta=\frac{p_{1}^{2} \mathrm{e}^{-\theta p_{2}}\left(p_{2} \lambda_{\mathrm{f}}-1\right)^{4}-p_{2}^{2} \mathrm{e}^{-\theta p_{1}}\left(p_{1} \lambda_{\mathrm{f}}-1\right)^{4}-p_{1}^{2}+p_{2}^{2}}{p_{1} p_{2}\left(p_{2}-p_{1}\right)}
\end{aligned}
$$

For the case that $\xi=1$, by substituting (22) into (19) and
(21), respectively, we obtain

$$
\begin{aligned}
\alpha= & \frac{1}{\omega_{\mathrm{n}}^{2}}\left[1+\mathrm{e}^{-\omega_{\mathrm{n}} \theta}\left(\omega_{\mathrm{n}} \lambda_{\mathrm{f}}-1\right)^{3}\right. \\
& \left.\times\left(1+\omega_{\mathrm{n}} \theta+3 \omega_{\mathrm{n}} \lambda_{\mathrm{f}}-\omega_{\mathrm{n}}^{2} \theta \lambda_{\mathrm{f}}\right)\right] \\
\beta= & \frac{1}{\omega_{\mathrm{n}}}\left[2+\mathrm{e}^{-\omega_{n} \theta}\left(\omega_{\mathrm{n}} \lambda_{\mathrm{f}}-1\right)^{3}\right. \\
& \left.\times\left(2+\omega_{\mathrm{n}} \theta+2 \omega_{\mathrm{n}} \lambda_{\mathrm{f}}-\omega_{\mathrm{n}}^{2} \theta \lambda_{\mathrm{f}}\right)\right]
\end{aligned}
$$

Thereby, it is seen that both $\alpha$ and $\beta$ are functions of $\lambda_{\mathrm{f}}$. So, there is still a single adjustable parameter, $\lambda_{\mathrm{f}}$, in the proposed IMC filter. It follows that

$$
F_{\mathrm{RIMC}-2}=\frac{\left(\alpha s^{2}+\beta s+1\right)\left(s^{2}+2 \xi \omega_{\mathrm{n}} s+\omega_{\mathrm{n}}^{2}\right)}{k \omega_{\mathrm{n}}^{2}\left(\lambda_{\mathrm{f}} s+1\right)^{4}}
$$

By substituting (16) and (22) into (2) and taking the inverse Laplace transform, the time response to a step change of $d_{\mathrm{i}}$ can be derived from

$y_{\mathrm{d}_{\mathrm{i}}}(t)=L^{-1}\left\{\frac{k \omega_{\mathrm{n}}^{2} \mathrm{e}^{-\theta s}}{s\left(s^{2}+2 \xi \omega_{\mathrm{n}} s+\omega_{\mathrm{n}}^{2}\right)}\left[1-\frac{\alpha s^{2}+\beta s+1}{\left(\lambda_{\mathrm{f}} s+1\right)^{4}} \mathrm{e}^{-\theta s}\right]\right\}$

In view of that DP cannot be analytically solved from (28), numerical guidelines are explored herein to disclose the quantitative tuning relationship between DP and $\lambda_{\mathrm{f}}$. By using the scaled complex variable, $\widehat{s}=s /\left(\xi \omega_{\mathrm{n}}\right)$, it can be verified from (28) that, given the values of $\lambda_{\mathrm{f}}\left(\xi \omega_{\mathrm{n}}\right)$ and $\theta\left(\xi \omega_{\mathrm{n}}\right), \mathrm{DP} / k$ is determined only by $\xi$, regardless of $\omega_{\mathrm{n}}$. By sweeping over the regions of $\lambda_{\mathrm{f}}\left(\xi \omega_{\mathrm{n}}\right) \in[0.2,2]$ and $\theta\left(\xi \omega_{\mathrm{n}}\right) \in[0.1,2]$, the numerical results for the three cases of $\xi=0.5, \xi=1.0$ and $\xi=1.5$ are plotted in Fig. 3, respectively. It can be seen that $\mathrm{DP} / k$ becomes larger as $\xi$ becomes smaller. Fig. $3 a$ indicates that the admissible tuning range of $\lambda_{\mathrm{f}}$ will be severely narrowed when $\xi$ is small. Figs. $3 b$ and $3 c$ show that $\mathrm{DP} / k$ increases monotonically with respect to $\lambda_{\mathrm{f}}\left(\xi \omega_{\mathrm{n}}\right)$ and $\theta\left(\xi \omega_{\mathrm{n}}\right)$, respectively. Note that DP for other values of $\xi$ may be quantitatively assessed using a linear interpolation method.

For the case that the load disturbance $\hat{d}_{\mathrm{o}}$ affects the process output with a first-order transfer function as often modelled for simplicity in engineering practices

$$
G_{\mathrm{d}}=\frac{k_{\mathrm{d}}}{\tau_{\mathrm{d}} s+1} \mathrm{e}^{-\theta_{\mathrm{d}} s}
$$

it can be derived from Fig. 1 that

$$
\frac{y_{\mathrm{di}}}{\hat{d}_{\mathrm{o}}}=G_{\mathrm{d}}(1-T)
$$

To reduce the influence arising from the time constant of $G_{\mathrm{d}}$ to the load disturbance response, the IMC filter is 

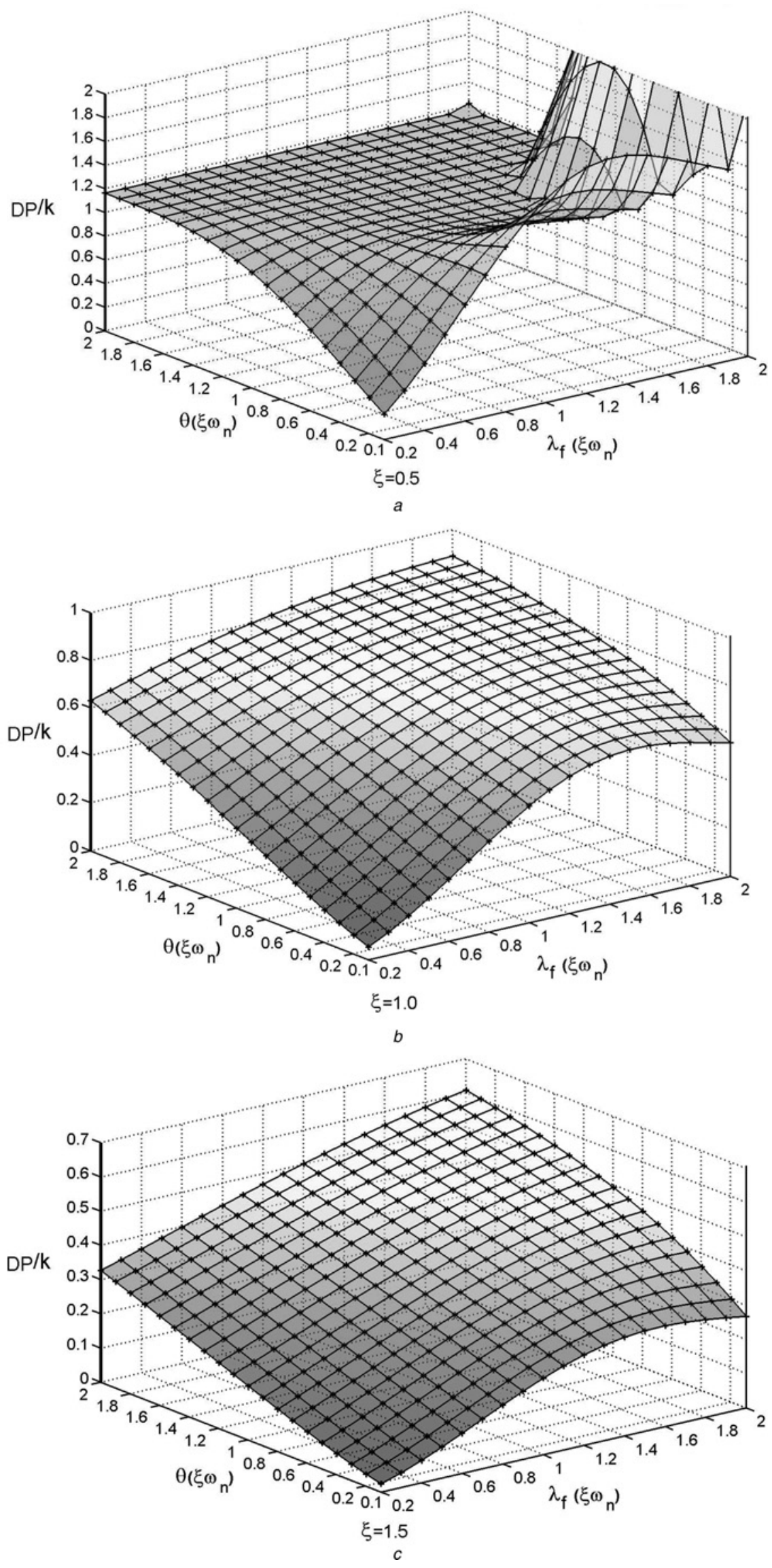

Figure $3 D P$ for a second-order process with

$a \xi=0.5 \quad b \xi=1.0 \quad c \xi=1.5$ 
rectified accordingly as

$$
f_{\mathrm{RIMC}-3}=\frac{\alpha s+1}{\left(\lambda_{\mathrm{f}} s+1\right)^{3}}
$$

where $\alpha$ is utilised to satisfy the following asymptotic constraint

$$
\lim _{s \rightarrow-1 / \tau_{\mathrm{d}}}(1-T)=0
$$

Note that the closed-loop complementary sensitivity function is therefore obtained as

$$
T_{\mathrm{RIMC}-3}=\frac{\alpha s+1}{\left(\lambda_{\mathrm{f}} s+1\right)^{3}} \mathrm{e}^{-\theta s}
$$

Substituting (33) into (32) yields

$$
\alpha=\tau_{\mathrm{d}}\left[1+\left(\frac{\lambda_{\mathrm{f}}}{\tau_{\mathrm{d}}}-1\right)^{3} \mathrm{e}^{-\theta / \tau_{\mathrm{d}}}\right]
$$

Accordingly, the feedback controller can be derived as

$$
F_{\mathrm{RIMC}-3}=\frac{(\alpha s+1)\left(s^{2}+2 \xi \omega_{\mathrm{n}} s+\omega_{\mathrm{n}}^{2}\right)}{k \omega_{\mathrm{n}}^{2}\left(\lambda_{\mathrm{f}} s+1\right)^{3}}
$$

It can be seen from (33) and (34) that $\mathrm{DP} / \mathrm{k}_{\mathrm{d}}$ is related to $\lambda_{\mathrm{f}} / \tau_{\mathrm{d}}$ and $\theta / \tau_{\mathrm{d}}$, regardless of the time delay, $\theta_{\mathrm{d}}$. By sweeping over the regions of $\lambda_{\mathrm{f}} / \tau_{\mathrm{d}} \in[0.2,2]$ and $\theta / \tau_{\mathrm{d}} \in[0.1,2]$, the numerical tuning guidelines are given in Fig. 4.

\section{Robust tuning constraints}

For a single-input-single-output (SISO) system, it is practical to lump multiple sources of process uncertainty into a multiplicative form to deal with [23]. According to the standard $M-\Delta$ structure for robustness analysis [24], the transfer function connecting the input and output of the process multiplicative uncertainty can be derived in terms of the closed-loop structure shown in Fig. 1, which is exactly equivalent to the closed-loop complementary sensitivity function, T. Hence, it follows from the small gain theorem that the perturbed closed-loop structure with the process multiplicative uncertainty holds robust stability if and only if

$$
\|T\|_{\infty}<\frac{1}{\|\Delta\|_{\infty}}
$$

where $\Delta=\left(G-G_{\mathrm{m}}\right) / G_{\mathrm{m}}$ denotes the process multiplicative uncertainty.

Note that for a SISO system, there exist $\|T\|_{\infty}=\sup (|T(j \omega)|)$ and $\|\Delta\|_{\infty}=\sup (|\Delta(j \omega)|), \quad \forall \omega \in$ $[0,+\infty)$. Denote $|\Delta|_{\mathrm{m}}=\sup (|\Delta(j \omega)|)$ for the convenience of analysis detailed in the following two subsections, respectively, for first- and second-order processes.

\subsection{Tuning constraints for first-order process}

It follows from (9) that

$$
|T(j \omega)|=\frac{\sqrt{\alpha^{2} \omega^{2}+1}}{\lambda_{\mathrm{f}}^{2} \omega^{2}+1}
$$

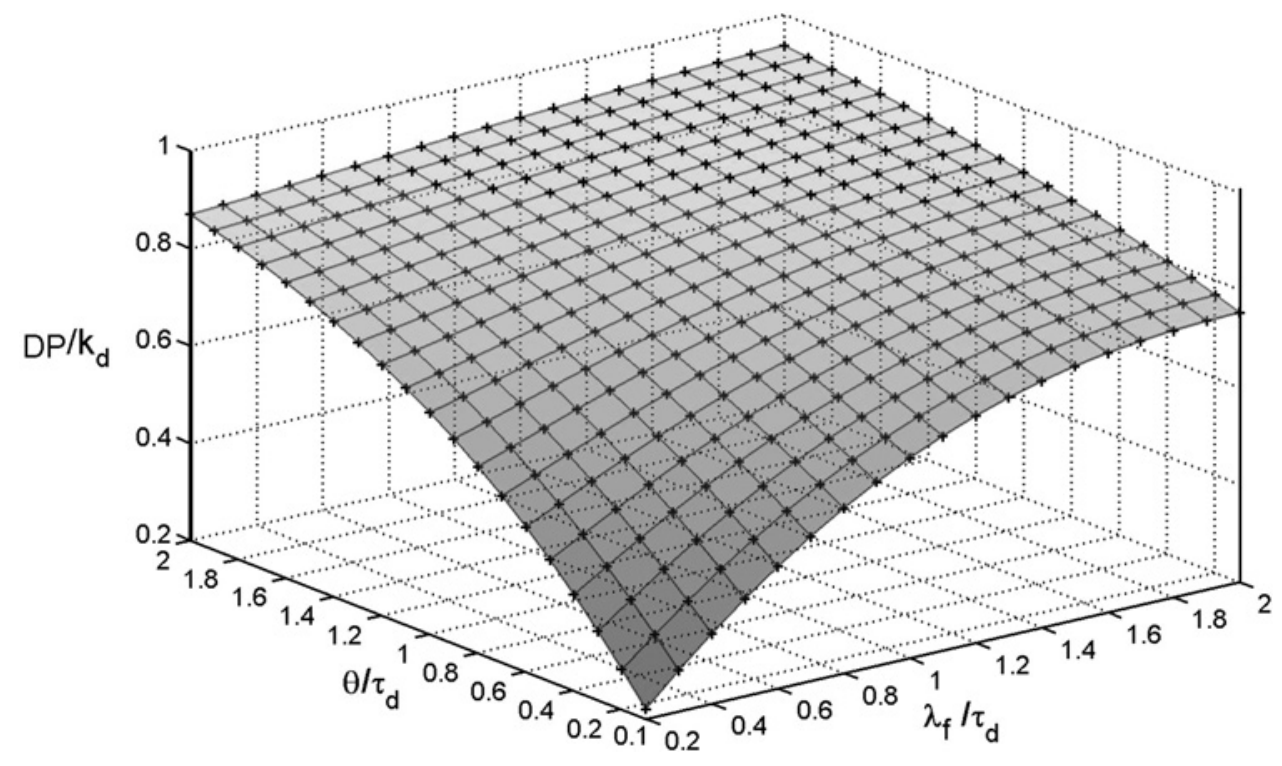

Figure $4 D P$ for a first-order load disturbance transfer function 
The first derivative can be obtained as

$$
\frac{\mathrm{d}|T(j \omega)|}{\mathrm{d} \omega}=\frac{\omega\left(\alpha^{2}-2 \lambda_{\mathrm{f}}^{2}-\alpha^{2} \lambda_{\mathrm{f}}^{2} \omega^{2}\right)}{\left(\lambda_{\mathrm{f}}^{2} \omega^{2}+1\right)^{2} \sqrt{\alpha^{2} \omega^{2}+1}}
$$

It can be verified by solving $\mathrm{d}|T(j \omega)| / \mathrm{d} \omega=0$ that $\omega=0$ is the unique extreme point to reach $\sup (|T(j \omega)|)$ for $\lambda_{\mathrm{f}} \geq \alpha / \sqrt{2}$, that is, $\sup (|T(j \omega)|)=|T(0)|=1$.

For $0<\lambda_{\mathrm{f}}<\alpha / \sqrt{2}$, there are two extreme points $\omega_{1}=0$ and $\omega_{2}=\sqrt{\alpha^{2}-2 \lambda_{\mathrm{f}}^{2}} /\left(\alpha \lambda_{\mathrm{f}}\right)$. Substituting the latter into (37) yields

$$
\left|T\left(j \omega_{2}\right)\right|=\frac{\alpha^{2}}{2 \lambda_{\mathrm{f}} \sqrt{\alpha^{2}-2 \lambda_{\mathrm{f}}^{2}}}
$$

It can be concluded from $\left(\alpha^{2}-2 \lambda_{\mathrm{f}}^{2}\right)^{2}>0$ that $\left|T\left(j \omega_{2}\right)\right|>1$. Therefore it follows that

$$
\sup (|T(j \omega)|)= \begin{cases}1 & \lambda_{\mathrm{f}} \geq \alpha / \sqrt{2} ; \\ \frac{\alpha^{2}}{2 \lambda_{\mathrm{f}} \sqrt{\alpha^{2}-2 \lambda_{\mathrm{f}}^{2}}} & 0<\lambda_{\mathrm{f}}<\alpha / \sqrt{2}\end{cases}
$$

To make clear the robust stability constraint to the tuning of $\lambda_{\mathrm{f}}$, the following proposition is given:

Proposition 2: Only a positive real root, $\lambda_{\mathrm{f}}=(\tau / 2)$ $\left[2-(1 / \varepsilon) \mathrm{e}^{(\theta / \tau)}+\sqrt{\left(2-(1 / \varepsilon) \mathrm{e}^{(\theta / \tau)}\right)^{2}+4\left(\mathrm{e}^{\theta / \tau}-1\right)}\right]$, exists for the equation of $\lambda_{\mathrm{f}}=\varepsilon \alpha$, that is, $\lambda_{\mathrm{f}}=$ $\varepsilon \tau\left[1-\left(\lambda_{\mathrm{f}} / \tau-1\right)^{2} \mathrm{e}^{-\theta / \tau}\right], \quad \forall \varepsilon>0, \quad$ which increases monotonically with respect to $\varepsilon$.

\section{Proof: See Appendix 1.}

For $\lambda_{\mathrm{f}} \geq \alpha / \sqrt{2}$, it can be solved using Proposition 2 that

$$
\lambda_{\mathrm{f}} \geq \frac{\tau}{2}\left[2-\sqrt{2} \mathrm{e}^{\theta / \tau}+\sqrt{\left(2-\sqrt{2} \mathrm{e}^{\theta / \tau}\right)^{2}+4\left(\mathrm{e}^{\theta / \tau}-1\right)}\right]
$$

Accordingly, it can be seen from (39) that once $\lambda_{\mathrm{f}}$ is increased into this region, the upper bound of $|T(j \omega)|$ is fixed as the unity, such that $|\Delta|_{\mathrm{m}}<1$ must be required for the closed-loop stability, regardless of the tuning of $\lambda_{\mathrm{f}}$ in this region. In other words, tuning $\lambda_{\mathrm{f}}$ in this region will not affect the permissible upper bound of $|\Delta|_{\mathrm{m}}$.

For $0<\lambda_{\mathrm{f}}<\alpha / \sqrt{2}$, it follows from Proposition 2 that

$$
\lambda_{\mathrm{f}}<\frac{\tau}{2}\left[2-\sqrt{2} \mathrm{e}^{\theta / \tau}+\sqrt{\left(2-\sqrt{2} \mathrm{e}^{\theta / \tau}\right)^{2}+4\left(\mathrm{e}^{\theta / \tau}-1\right)}\right]
$$

Combining (36) with (39), the robust stability constraint can be determined as

$$
\frac{\alpha^{2}}{2 \lambda_{\mathrm{f}} \sqrt{\alpha^{2}-2 \lambda_{\mathrm{f}}^{2}}}<\frac{1}{|\Delta|_{\mathrm{m}}}
$$

It follows from solving (42) that

$$
\sqrt{\frac{1-\sqrt{1-|\Delta|_{\mathrm{m}}^{2}}}{2}} \alpha<\lambda_{\mathrm{f}}<\sqrt{\frac{1+\sqrt{1-|\Delta|_{\mathrm{m}}^{2}}}{2}} \alpha
$$

Denote

$$
\eta=\sqrt{\frac{1-\sqrt{1-|\Delta|_{\mathrm{m}}^{2}}}{2}}, \quad \gamma=\sqrt{\frac{1+\sqrt{1-|\Delta|_{\mathrm{m}}^{2}}}{2}}
$$

Using Proposition 2 to solve (43), we obtain

$$
\begin{gathered}
\frac{\tau}{2}\left[2-\frac{1}{\eta} \mathrm{e}^{\theta / \tau}+\sqrt{\left(2-\frac{1}{\eta} \mathrm{e}^{\theta / \tau}\right)^{2}+4\left(\mathrm{e}^{\theta / \tau}-1\right)}\right]<\lambda_{\mathrm{f}} \\
<\frac{\tau}{2}\left[2-\frac{1}{\gamma} \mathrm{e}^{\theta / \tau}+\sqrt{\left(2-\frac{1}{\gamma} \mathrm{e}^{\theta / \tau}\right)^{2}+4\left(\mathrm{e}^{\theta / \tau}-1\right)}\right]
\end{gathered}
$$

Note that $\eta<1 / \sqrt{2}$ and $\gamma>1 / \sqrt{2}$. Comparing (41) and (44), we obtain the robust tuning constraint

$$
\begin{gathered}
\frac{\tau}{2}\left[2-\frac{1}{\eta} \mathrm{e}^{\theta / \tau}+\sqrt{\left(2-\frac{1}{\eta} \mathrm{e}^{\theta / \tau}\right)^{2}+4\left(\mathrm{e}^{\theta / \tau}-1\right)}\right]<\lambda_{\mathrm{f}} \\
<\frac{\tau}{2}\left[2-\sqrt{2} \mathrm{e}^{\theta / \tau}+\sqrt{\left(2-\sqrt{2} \mathrm{e}^{\theta / \tau}\right)^{2}+4\left(\mathrm{e}^{\theta / \tau}-1\right)}\right]
\end{gathered}
$$

Remark 3: For the process gain uncertainty, $\Delta k$, the robust stability constraint of (36) is equivalent to the Nyquist stability criterion, $|T(j \omega)|<1 /|\Delta(j \omega)|$, since $\Delta k \in \mathbb{R}$. However, the above robust tuning constraint of (45) may be somewhat conservative for other process uncertainties. For instance, parameter uncertainties of the time constant and time delay are far less likely to result in a phase change over $-\pi$ in practice, so $\sup (|T(j \omega)|)<1 /|\Delta|_{\mathrm{m}}$ is not necessary if $|T(j \omega)|<1 /|\Delta(j \omega)|$ is satisfied for $\omega \in[0, \infty)$.

\subsection{Tuning constraints for second-order process}

It follows from (22) that

$$
|T(j \omega)|=\frac{\sqrt{\left(\alpha^{2} \omega^{2}-1\right)^{2}+\beta^{2} \omega^{2}}}{\left(\lambda_{\mathrm{f}}^{2} \omega^{2}+1\right)^{2}}
$$


The first derivative can be obtained as

$$
\begin{aligned}
\frac{\mathrm{d}|T(j \omega)|}{\mathrm{d} \omega}= & \frac{\omega}{\left(\lambda_{\mathrm{f}}^{2} \omega^{2}+1\right)^{3}}\left[\frac{2 \alpha\left(\alpha \omega^{2}-1\right)+\beta^{2}}{\sqrt{\left(\alpha \omega^{2}-1\right)^{2}+\beta^{2} \omega^{2}}}\left(\lambda_{\mathrm{f}}^{2} \omega^{2}+1\right)\right. \\
& \left.-4 \lambda_{\mathrm{f}}^{2} \sqrt{\left(\alpha \omega^{2}-1\right)^{2}+\beta^{2} \omega^{2}}\right]
\end{aligned}
$$

Obviously, $\omega=0$ is an extreme point corresponding to $|T(0)|=1$. For $\omega \neq 0$, it follows from $\mathrm{d}|T(j \omega)| / \mathrm{d} \omega=0$ that

$$
2 \alpha^{2} \lambda_{\mathrm{f}}^{2} x^{2}+\left[3 \lambda_{\mathrm{f}}^{2}\left(\beta^{2}-2 \alpha\right)-2 \alpha^{2}\right] x+4 \lambda_{\mathrm{f}}^{2}+2 \alpha-\beta^{2}=0
$$

where $x=\omega^{2}$. Denote $A_{0}=2 \alpha^{2} \lambda_{\mathrm{f}}^{2}, B_{0}=3 \lambda_{\mathrm{f}}^{2}\left(\beta^{2}-2 \alpha\right)-2 \alpha^{2}$ and $C_{0}=4 \lambda_{\mathrm{f}}^{2}+2 \alpha-\beta^{2}$, the quadratic discriminant of (48) can be expressed as $\delta_{0}=B_{0}^{2}-4 A_{0} C_{0}$. Correspondingly, the robust constraint to the tuning of $\lambda_{\mathrm{f}}$ is given in the following proposition.

Proposition 3: For a second-order process described in (16), the closed-loop structure shown in Fig. 1 for load disturbance rejection holds robust stability if and only if $\sup (|T(j \omega)|)<1 /|\Delta|_{\mathrm{m}}$, where (see equation at the bottom of the page)

$$
\begin{gathered}
\omega_{1}=\sqrt{\frac{-B_{0}+\sqrt{B_{0}^{2}-4 A_{0} C_{0}}}{2 A_{0}}} \text { and } \\
\omega_{2}=\sqrt{\frac{-B_{0}-\sqrt{B_{0}^{2}-4 A_{0} C_{0}}}{2 A_{0}}}
\end{gathered}
$$

Proof: See Appendix 2.

For the case to reject $\hat{d}_{0}$ from the process output side with a first-order transfer function, it follows from (33) that

$$
|T(j \omega)|=\frac{\sqrt{\alpha^{2} \omega^{2}+1}}{\left(\lambda_{\mathrm{f}}^{2} \omega^{2}+1\right)^{3 / 2}}
$$

The first derivative can be derived as

$$
\frac{\mathrm{d}|T(j \omega)|}{\mathrm{d} \omega}=\frac{\omega}{\left(\lambda_{\mathrm{f}}^{2} \omega^{2}+1\right)^{5 / 2}}\left[\frac{\alpha^{2}\left(\lambda_{\mathrm{f}}^{2} \omega^{2}+1\right)}{\sqrt{\alpha^{2} \omega^{2}+1}}-3 \lambda_{\mathrm{f}}^{2} \sqrt{\alpha^{2} \omega^{2}+1}\right]
$$

Note that $\omega=0$ is an obvious extreme point corresponding to $|T(0)|=1$. The robust constraint to tuning $\lambda_{\mathrm{f}}$ is given in the following proposition.

Proposition 4: For a second-order process described in (16), the closed-loop structure for rejecting load disturbances from the process output side with a first-order transfer function holds robust stability if and only if $|\Delta|_{\mathrm{m}}<1$.

\section{Proof: See Appendix 3.}

Remark 4: It is implied by Proposition 4 that the tuning of $\lambda_{\mathrm{f}}$ does not affect the closed-loop robust stability since $\sup (|T(j \omega)|)=1$ for $\lambda_{\mathrm{f}} \in(0,+\infty)$. Hence, tuning $\lambda_{\mathrm{f}}$ can be focused purely on the desired closed-loop performance for rejecting such load disturbance.

\section{Illustration}

Example 1: Consider the slow process with time delay

$$
G=\frac{\mathrm{e}^{-30 s}}{100 s+1}
$$

A conventional IMC filter should determine the feedback controller shown in Fig. 1 for load disturbance rejection as $F_{\text {IMC }}=(100 s+1) /(\lambda s+1)$. Using the proposed IMC filter shown in (7), it follows from the proposed formula of (11) that

$$
F_{\mathrm{RIMC}-1}=\frac{(\alpha s+1)(100 s+1)}{\left(\lambda_{\mathrm{f}} s+1\right)^{2}}
$$

where $\alpha=100\left[1-\left(0.01 \lambda_{\mathrm{f}}-1\right)^{2} \mathrm{e}^{-0.3}\right]$. For comparison, adding a unit step change of $d_{\mathrm{i}}$ to the process and taking $\lambda=\lambda_{\mathrm{f}}=40$, we obtain the output responses shown in Fig. 5. It is seen that obviously improved load disturbance rejection is obtained by the proposed IMC filter. The conventional IMC filter has led to a long 'tail' in the load disturbance response due to the influence resulting from the slow time constant of the process. To obtain the same DP with the proposed IMC filter, $\lambda=20$ is required for the conventional IMC filter, as shown in Fig. 5, but the recovery time is still about $50 \%$ longer.

Assume that there exists $30 \%$ error in the process parameter estimation. The worst case is that the process time constant is actually 30\% smaller and the time delay $30 \%$ larger. The corresponding output responses are shown

$$
\sup (|T(j \omega)|)= \begin{cases}\max \left\{1,\left|T\left(j \omega_{1}\right)\right|\right\} & \delta_{0}>0, C_{0} \leq 0 ; \\ \max \left\{1,\left|T\left(j \omega_{1}\right)\right|,\left|T\left(j \omega_{2}\right)\right|\right\} & \delta_{0}>0, B_{0}<0, C_{0}>0 ; \\ \max \left\{1,\left|T\left(j \sqrt{\frac{-B_{0}}{2 A_{0}}}\right)\right|\right\} & \delta_{0}=0, B_{0}<0 ; \\ 1 & \text { else }\end{cases}
$$



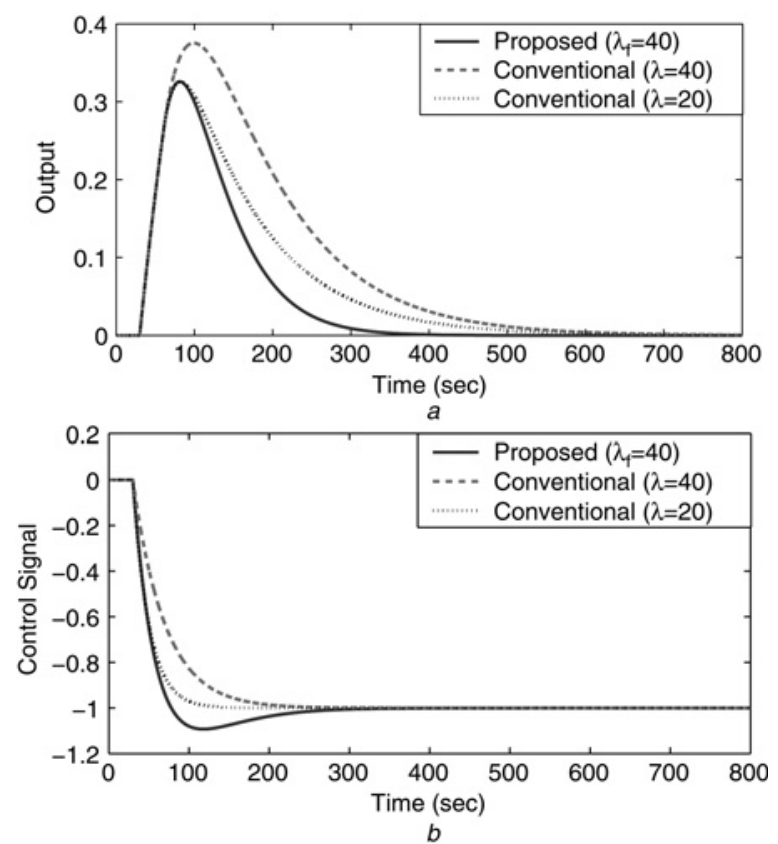

Figure 5 Nominal output responses for Example 1

in Fig. 6, demonstrating that the proposed IMC filter performs better against the severe process uncertainties.

Example 2: Consider the second-order process studied in the recent paper [8]

$$
G=\frac{\mathrm{e}^{-s}}{(20 s+1)(2 s+1)}
$$

Skogestad [8] derived a IMC-based PID controller, $C=10(0.125 / s+1)(2 s+1), \quad$ to optimise the system
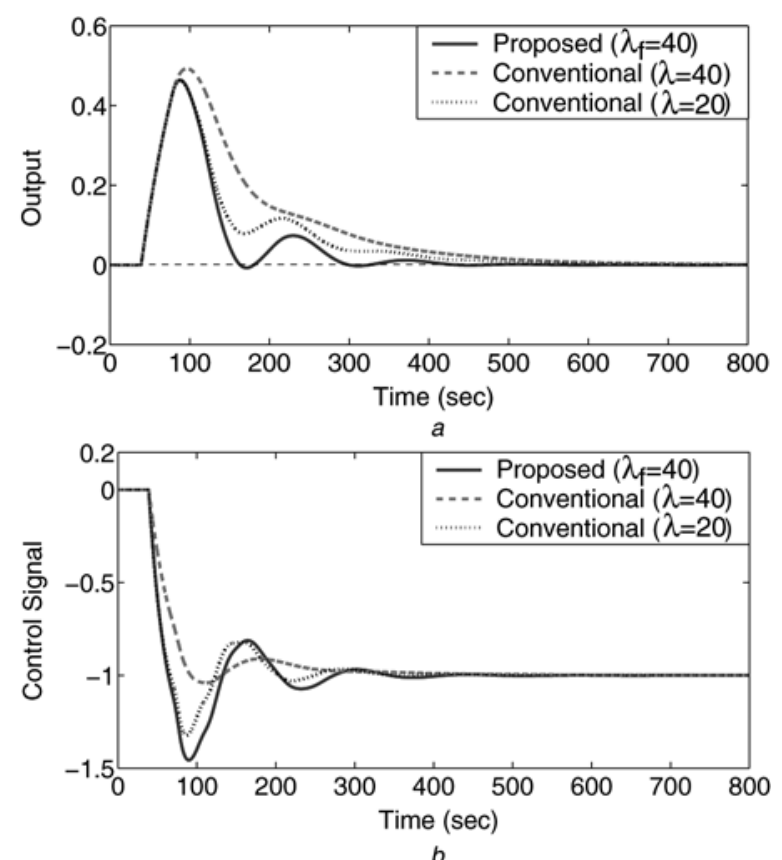

Figure 6 Perturbed output responses for Example 1 performance against load disturbance, of which the adjustable parameter, $\tau_{\mathrm{c}}=1.0$, corresponds to $\lambda=1.0$ in the conventional IMC filter shown in (17). The feedback controller $F$ in Fig. 1 should be configured as $F_{\text {IMC }}=(20 s+1)(2 s+1) /(\lambda s+1)^{2}$ according to the conventional IMC filter. Using the proposed IMC filter shown in (18), it follows from (23), (24) and (27) that

$$
F_{\mathrm{RIMC}-2}=\frac{\left(\alpha s^{2}+\beta s+1\right)(20 s+1)(2 s+1)}{\left(\lambda_{\mathrm{f}} s+1\right)^{4}}
$$

where

$$
\begin{gathered}
\alpha=2.6957\left(0.5 \lambda_{\mathrm{f}}-1\right)^{4}-42.2769\left(0.05 \lambda_{\mathrm{f}}-1\right)^{4}+40, \\
\beta=0.1348\left(0.5 \lambda_{\mathrm{f}}-1\right)^{4}-21.1384\left(0.05 \lambda_{\mathrm{f}}-1\right)^{4}+22 .
\end{gathered}
$$

For comparison, adding a unit step change of $d_{\mathrm{i}}$ to the process and taking $\lambda=\lambda_{\mathrm{f}}=1.0$, we obtain the output responses shown in Fig. 7. It is again seen that apparently improved load disturbance response is obtained using the proposed IMC filter. To obtain the same DP with the proposed IMC filter, $\lambda=0.09$ and $\tau_{\mathrm{c}}=0.15$ are required respectively for the conventional IMC filter and the IMCbased controller of Skogestad [8]. It is seen that the recovery time cannot be efficiently reduced by using the conventional IMC filter, whereas the controller of Skogestad [8] yields an oscillatory response. Moreover, it can be verified that, if the process time delay is actually $20 \%$ larger and the slower time constant $\left(\tau_{1}=20\right)$ is $20 \%$ smaller, the proposed IMC filter maintains well the closedloop stability, whereas the conventional IMC filter with $\lambda=0.09$ cannot hold the closed-loop stability any longer, and the IMC-based controller of Skogestad [8] yields very oscillatory response.

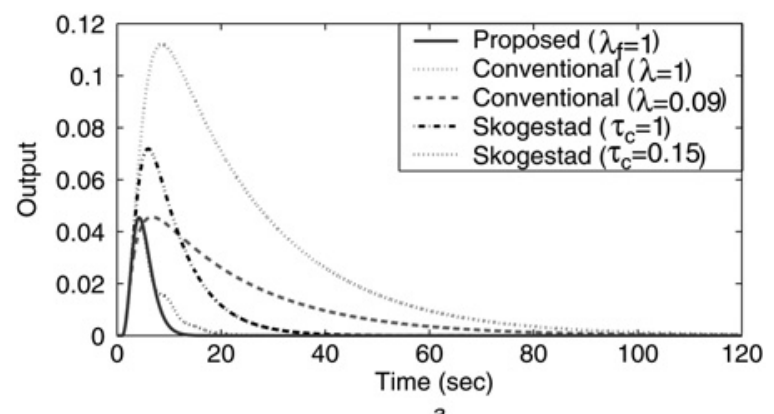

a

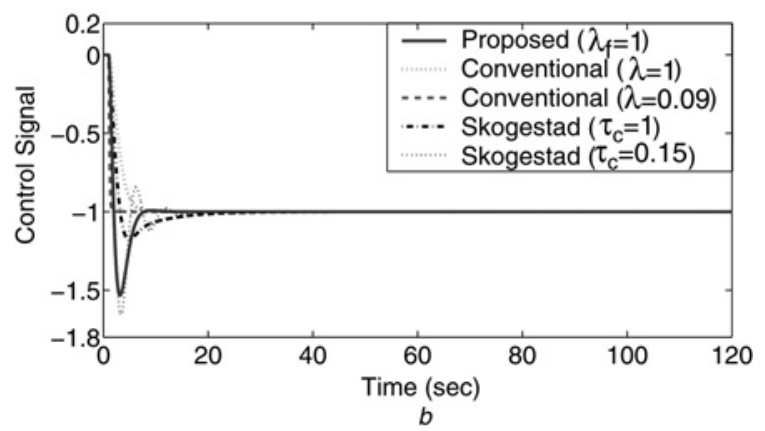

Figure 7 Nominal output responses for Example 2 
To demonstrate the achievable performance for rejecting load disturbance from the process output side (denoted as $\hat{d}_{0}$ in Fig. 1) with slow dynamics, for example, $G_{\mathrm{d}}=\mathrm{e}^{-2 s} /(10 s+1)$, the proposed IMC filter shown in (31) should be used to design the feedback controller as

$$
F_{\mathrm{RIMC}-3}=\frac{(\alpha s+1)(20 s+1)(2 s+1)}{\left(\lambda_{\mathrm{f}} s+1\right)^{3}}
$$

where $\alpha=10\left[1+\left(0.1 \lambda_{\mathrm{f}}-1\right)^{3} \mathrm{e}^{-0.1}\right]$. Assume that there exists $50 \%$ error for estimating the time constant of the load disturbance transfer function. The worst case is that the time constant is actually $50 \%$ smaller. Note that the time delay, $\theta_{\mathrm{d}}=2$, of the load disturbance transfer function does not affect the disturbance response performance as discussed in the filter design procedure in Section 2.2. By adding a unit step change of $\hat{d}_{0}$, and taking $\lambda=\lambda_{\mathrm{f}}=1.0, \lambda=0.45$ and $\tau_{\mathrm{c}}=0.6$ for comparison, we obtain the output responses shown in Fig. 8. It is seen that the proposed IMC filter results in apparently improved disturbance rejection, given the severe time constant error of modelling the load disturbance transfer function.

\section{Conclusions}

Filter design is a key in the IMC theory for closed-loop controller tuning. This paper has proposed a modified IMC filter design to improve controller tuning for load disturbance rejection. The idea lies with countering the influence resulting from the slow time constant(s) of the process or repetitive-type load disturbance by establishing asymptotic canceling constraints. Based on the widely used

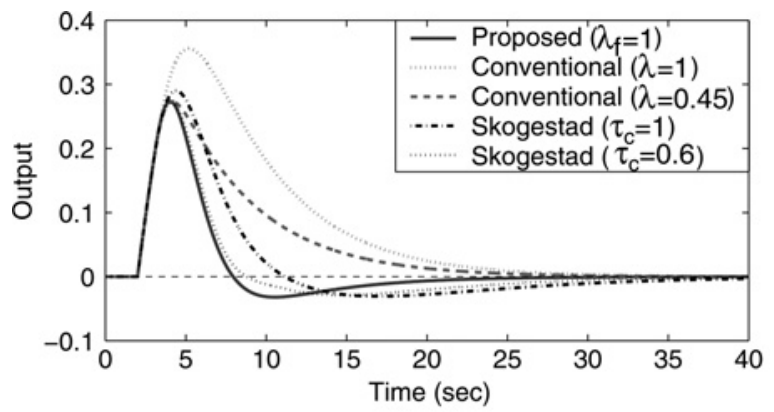

a

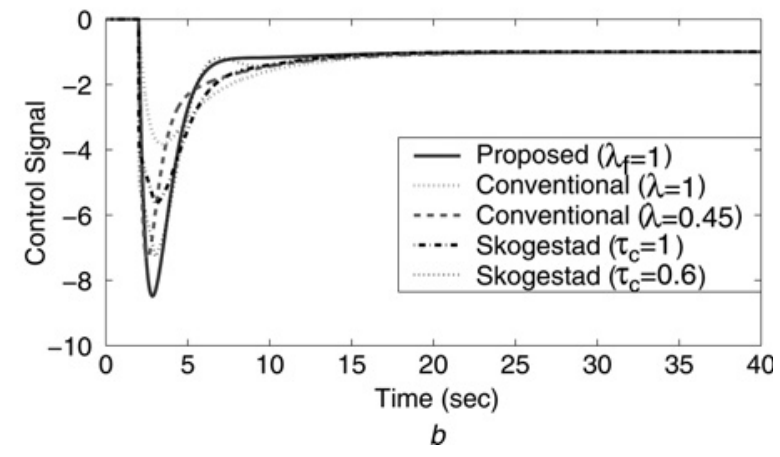

Figure 8 Output responses of Example 2 for the presence of a slow load disturbance first- and second-order time delay process models, analytical formulae and quantitative guidelines have been developed for tuning the single adjustable parameter of the proposed IMC filter, in terms of the commonly used disturbance rejection specifications, recovery time and DP, for assessment of disturbance response performance in engineering practices. Note that the proposed IMC filter design and the corresponding robust stability analysis can be directly applied to tune an IMC-based controller in the unity feedback control structure, and also can be transparently extended to higher order processes. Illustrative examples have demonstrated that the proposed IMC filter can result in noteworthy improvement of disturbance rejection performance for slow processes or for the presence of repetitive-type load disturbance with slow dynamics.

\section{Acknowledgment}

This work is supported in part by Hong Kong Research Grant Council under Project No.613107 and China National 973 Program (2009CB320603).

\section{References}

[1] MORARI M., ZAFIRIOU E.: 'Robust process control' (Prentice-Hall, New Jersey, 1989)

[2] Ogunnaike B.A., RAY W.H.: 'Process dynamics, modeling, and control' (Oxford University Press, New York, 1994)

[3] BRAATZ R.D.: 'Internal model control: the control handbook' (CRC Press, Boca Raton, FL, 1995)

[4] Seborg D.E., edgar T.F., MEllichamp D.A.: 'Process dynamics and control' (Wiley, Hoboken, NJ, 2004, 2nd edn.)

[5] CHIEN I.L., FRUEhAUF P.S.: 'Consider IMC tuning to improve controller performance', Chem. Eng. Prog., 1990, 86, (1), pp. 33-41

[6] WANG Q.G., YANG X.P.: 'Single-loop controller design via IMC principles', Automatica, 2001, 37, (12), pp. 2041-2048

[7] LEE Y., PARK S., LEE M., BROSILOW C.: 'PID controller tuning for desired closed-loop responses for SI/SO systems', AIChE J., 1998, 44, (1), pp. 106-115

[8] SKOgESTAD S.: 'Simple analytical rules for model reduction and PID controller tuning', J. Process Control, 2003, 13, (4), pp. 291-309

[9] LEVA A.: 'Model-based proportional-integral-derivative autotuning improved with relay feedback identification', IEE Proc. Control Theory Appl., 2005, 152, (2), pp. $247-256$ 
[10] LIU T., GAO F.: 'Relay-based autotuning of PID controller for improved load disturbance rejection'. 17th IFAC World Congress, Seoul, Korea, 2008, vol. 4, pp. 10933-10938

[11] LEE J., EDGAR T.F.: 'ISE tuning rule revisited', Automatica, 2004, 40, (8), pp. 1455-1458

[12] JOSE A.R., ROSENDO M.L., ALEJANDRA V.: 'Optimality of internal model control tuning rules', Ind. Eng. Chem. Res., 2004, 43, (24), pp. 7951-7958

[13] LIU T., ZHANG W.D., GU D.Y.: 'Analytical design of decoupling internal model control (IMC) scheme for two-input-twooutput (TITO) processes with time delays', Ind. Eng. Chem. Res., 2006, 45, (9), pp. 3149-3160

[14] TIAN Y., GAO F.: 'Double-controller scheme for control of processes with dominant delay', IEE Proc. Control Theory Appl., 1998, 145, (5), pp. 479-484

[15] ZHANG W.D., SUN Y.X., XU X.M.: 'Two degree-of-freedom Smith predictor for processes with time delay', Automatica, 1998, 34, (10), pp. 1279-1282

[16] NORMEY-RICO J.E., CAMACHO E.F.: 'A unified approach to design dead-time compensators for stable and integrative processes with dead-time', IEEE Trans. Autom. Control, 2003, 47, (2), pp. 299-305

[17] RIVERA D.E., MORARI M., SKOGESTAD S.: 'Internal model control. 4. PID controller design', Ind. Eng. Chem. Res., 1986, 25, (2), pp. 252-265

[18] HORN I.G., ARULANDU J.R., BRAATZ R.D.: 'Improved filter design in internal model control', Ind. Eng. Chem. Res., 1996, 35, (10), pp. 3437-3441

[19] SHAMSUZZOHA M., LEE M.: 'IMC-PID controller design for improved disturbance rejection of time-delayed processes', Ind. Eng. Chem. Res., 2007, 46, (7), pp. 2077-2091

[20] MIDDLETON R.H., GRAeBE S.F.: 'Slow stable open-loop poles: to cancel or not to cancel', Automatica, 1999, 35, (5), pp. $877-886$

[21] CAMPI M., LEE S.W., ANDERSON B.D.O.: 'New filters for internal model control design', Int. J. Robust Nonlinear Control, 1994, 4, (6), pp. 757-775

[22] HWANG C., CHENG Y.C.: 'A note on the use of the Lambert W function in the stability analysis of time-delay systems', Automatica, 2005, 41, (11), pp. 1979-1985

[23] SkOgestad S., PostlethWAite I.: 'Multivariable feedback control: analysis and design' (Wiley, Chichester, 2005, 2nd edn.)

[24] ZHOU K.M., DOYLE J.C., GLOVER K.: 'Robust and optimal control' (Prentice-Hall, Englewood Cliffs, NJ, 1996)

\section{Appendix 1: proof of Proposition 2}

Substituting $\quad \alpha=\tau\left[1-\left(\left(\lambda_{\mathrm{f}} / \tau\right)-1\right)^{2} \mathrm{e}^{-\theta / \tau}\right] \quad$ into the equation shown in Proposition 2, we obtain

$$
\left(\frac{\lambda_{\mathrm{f}}}{\tau}-1\right)^{2}+\frac{1}{\varepsilon} e^{\theta / \tau} \frac{\lambda_{\mathrm{f}}}{\tau}-e^{\theta / \tau}=0
$$

Then it can be derived from (50) that there exist two real roots

$$
\lambda_{\mathrm{f}-1,2}=\frac{\tau}{2}\left[2-\frac{1}{\varepsilon} \mathrm{e}^{\theta / \tau} \pm \sqrt{\left(2-\frac{1}{\varepsilon} \mathrm{e}^{\theta / \tau}\right)^{2}+4\left(\mathrm{e}^{\theta / \tau}-1\right)}\right]>0
$$

In view of that $\lambda_{\mathrm{f}}$ is an adjustable parameter of $F$, the negative real root should be abandoned.

Let

$$
f(\varepsilon)=2-\frac{1}{\varepsilon} \mathrm{e}^{\theta / \tau}+\sqrt{\left(2-\frac{1}{\varepsilon} \mathrm{e}^{\theta / \tau}\right)^{2}+4\left(\mathrm{e}^{\theta / \tau}-1\right)}
$$

the first derivative of $f(\varepsilon)$ can be derived as

$$
\frac{\mathrm{d} f(\varepsilon)}{\mathrm{d} \varepsilon}=\frac{1}{\varepsilon^{2}} \mathrm{e}^{\theta / \tau}\left[1+\frac{2-(1 / \varepsilon) \mathrm{e}^{\theta / \tau}}{\sqrt{\left(2-(1 / \varepsilon) \mathrm{e}^{\theta / \tau}\right)^{2}+4\left(\mathrm{e}^{\theta / \tau}-1\right)}}\right]>0
$$

Hence, $f(\varepsilon)$ increases monotonically with respect to $\varepsilon$, and so is for $\lambda_{\mathrm{f}}$ with respect to $\varepsilon$.

\section{Appendix 2: proof of Proposition 3}

There are six cases categorised for deriving $\sup (|T(j \omega)|)$. Note that $\delta_{0}>0$ is for cases (i) and (ii). Case (iv), 'else', includes three subcases that lead to no positive real solution of (48), that is, ' $\delta_{0}<0$ ', ' $\delta_{0}>0, B_{0}>0, C_{0}>0$ ' and ' $\delta_{0}=0, B_{0} \geq 0$ '.

For $\delta_{0}>0$, there are two real roots for (48), that is

$$
x_{1,2}=\frac{-B_{0} \pm \sqrt{B_{0}^{2}-4 A_{0} C_{0}}}{2 A_{0}}
$$

If $C_{0}<0$, that is, case (i), it can be seen that $\sqrt{\delta_{0}}>\left|B_{0}\right|$, corresponding to $x_{1}>0$ and $x_{2}<0$. Accordingly, there exists only one positive real root of $x=\omega^{2}$, that is, $\omega_{1}=\sqrt{x_{1}}$. So, $\sup (|T(j \omega)|)$ may be reached at $\omega=0$ or $\omega_{1}$. For case (ii), it can be seen that $\sqrt{\delta_{0}}<\left|B_{0}\right|$, corresponding to $x_{1}>0$ and $x_{2}>0$. Accordingly, there exist two positive real roots of $x=\omega^{2}$, that is, $\omega_{1}=\sqrt{x_{1}}$ 
and $\omega_{2}=\sqrt{x_{2}}$. So, $\sup (|T(j \omega)|)$ may be reached at $\omega=0$, $\omega_{1}$ or $\omega_{2}$.

For case (iii), it can be seen that (48) has a dual positive root at $x=-B_{0} / 2 A_{0}$. Correspondingly, $\sup (|T(j \omega)|)$ may be reached at $\omega=0$ or $\sqrt{-B_{0} / 2 A_{0}}$.

For the case that $\delta_{0}<0$, there is no real roots for (48) according to the solvability of a linear quadric equation. Thus, $\sup (|T(j \omega)|)$ can only be reached at $\omega=0$. This conclusion can be similarly drawn for the other two subcases of case (iv).

Hence, using the small gain theorem, we obtain the conclusion in Proposition 3.

\section{Appendix 3: proof of Proposition 4}

It follows from $\mathrm{d}|T(j \omega)| / \mathrm{d} \omega=0$ that

$$
\alpha^{2}\left(\lambda_{\mathrm{f}}^{2} \omega^{2}+1\right)-3 \lambda_{\mathrm{f}}^{2}\left(\alpha^{2} \omega^{2}+1\right)=0
$$

The solution of (51) can be derived as

$$
\omega^{2}=\frac{\alpha^{2}-3 \lambda_{f}^{2}}{2 \alpha^{2} \lambda_{f}^{2}}
$$

To obtain a positive solution of $\omega$, it requires that

$$
\lambda_{\mathrm{f}}<\frac{\alpha}{\sqrt{3}}
$$

The extreme point can thus be derived as

$$
\omega=\frac{\sqrt{\alpha^{2}-3 \lambda_{\mathrm{f}}^{2}}}{\sqrt{2} \alpha \lambda_{\mathrm{f}}}
$$

Substituting (54) into (49) yields

$$
|T(j \omega)|=\frac{2 \alpha^{3}}{3 \sqrt{3} \lambda_{\mathrm{f}}\left(\alpha^{2}-\lambda_{\mathrm{f}}^{2}\right)}
$$

Let $g\left(\lambda_{\mathrm{f}}\right)$ be a function with respect to $\lambda_{\mathrm{f}}$, that is

$$
g\left(\lambda_{\mathrm{f}}\right)=3 \sqrt{3} \lambda_{\mathrm{f}}\left(\alpha^{2}-\lambda_{\mathrm{f}}^{2}\right)
$$

The first derivative of $g\left(\lambda_{\mathrm{f}}\right)$ can be derived as

$$
g^{\prime}\left(\lambda_{\mathrm{f}}\right)=3 \sqrt{3}\left(\alpha^{2}-3 \lambda_{\mathrm{f}}^{2}\right)
$$

Combining (53) with (57), it can be seen that $g^{\prime}\left(\lambda_{\mathrm{f}}\right)>0$. Thus, $\lambda_{\mathrm{f}}=\alpha / \sqrt{3}$ is the unique extreme point to reach the maximum of $g\left(\lambda_{\mathrm{f}}\right)$, that is

$$
\max \left\{g\left(\lambda_{\mathrm{f}}\right)\right\}=3 \sqrt{3} \frac{\alpha}{\sqrt{3}}\left[\alpha^{2}-\left(\frac{\alpha}{\sqrt{3}}\right)^{2}\right]=2 \alpha^{3}
$$

Substituting (58) into (55) yields

$$
|T(j \omega)|<1, \quad \forall \omega>0
$$

Hence, $\sup (|T(j \omega)|)$ is reached only at $\omega=0$, that is, $\sup (|T(j \omega)|)=1$.

Using the small gain theorem, we conclude that $\sup |\Delta(j \omega)|<1$ is required to hold the closed-loop stability. 